ARTICLE

Received 7 May 2015 | Accepted 14 Jan 2016 | Published 8 Mar 2016

DOI: $10.1038 /$ ncomms10722

OPEN

\title{
Drift transport of helical spin coherence with tailored spin-orbit interactions
}

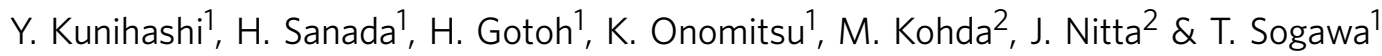

Most future information processing techniques using electron spins in non-magnetic semiconductors will require both the manipulation and transfer of spins without their coherence being lost. The spin-orbit effective magnetic field induced by drifting electrons enables us to rotate the electron spins in the absence of an external magnetic field. However, the fluctuations in the effective magnetic field originating from the random scattering of electrons also cause undesirable spin decoherence, which limits the length scale of the spin transport. Here we demonstrate the drift transport of electron spins adjusted to a robust spin structure, namely a persistent spin helix. We find that the persistent spin helix enhances the spatial coherence of drifting spins, resulting in maximized spin decay length near the persistent spin helix condition. Within the enhanced distance of the spin transport, the transport path of electron spins can be modulated by employing time-varying in-plane voltages.

\footnotetext{
${ }^{1}$ NTT Basic Research Laboratories, NTT Corporation, 3-1 Morinosato-Wakamiya, Atsugi, Kanagawa 243-0198, Japan. ${ }^{2}$ Department of Materials Science, Tohoku University, 6-6-02 Aramaki-Aza Aoba, Aoba-ku, Sendai 980-8579, Japan. Correspondence and requests for materials should be addressed to Y.K. (email: kunihashi.y@lab.ntt.co.jp).
} 
$\mathrm{T}$ ransporting electron spins in spin-orbit coupled systems is the key to developing future spintronics devices and spinbased information processing ${ }^{1-3}$. Conduction electrons moving in semiconductors experience the spin-orbit interaction (SOI) as an effective magnetic field, which enables us to rotate the electron spins in the absence of an external magnetic field. At the same time, the SOI also has an adverse effect: the fluctuations in the effective magnetic field originating from the random scattering of electrons also cause undesirable spin decoherence ${ }^{4}$, which limits the length scale of spin transport. The enhancement of spin coherence during drift transport is essential for spin-based devices but has yet to be demonstrated.

The two-dimensional electron systems in semiconductors are promising for manipulating travelling spins with controllable SOIs. This is not just because of their high mobility, which leads to high-speed spin transfer, but also because of the ability to tune the strength of the Rashba SOI by applying a vertical electric field. Furthermore, the tunability of the SOI also provides the chance to balance the Dresselhaus and Rashba SOIs. This condition results in a robust spin structure for spin-independent scattering, namely a persistent spin helix (PSH), which has a helical spin density wave with an infinite spin lifetime. The PSH has been theoretically predicted ${ }^{6,7}$ and experimentally demonstrated by measuring the lifetime of the helical spin mode generated by the transient spin grating technique ${ }^{8}$.

Here we demonstrate the drift transport of electron spins in the PSH state, which has so far only been studied without lateral electric fields ${ }^{8-10}$. Recently, Yang et al. ${ }^{11,12}$ observed the drift transport of helical spin structures under unbalanced Dresselhaus and Rashba SOI conditions. We expect that the balanced condition realized by careful tuning of the gate-controlled Rashba SOI will effectively extend the length of the drift spin transport. In our experiment, a multi-cycle spin precession was observed for a specific direction, while it was completely quenched for other orthogonal directions, reflecting the achievement of an exactly balanced SOI condition. A theoretical model reproduced the spin distributions well and revealed that the spin decay length is maximized near the PSH condition. Within the enhanced distance of the spin transport, the transport path of electron spins can be modulated by employing timevarying in-plane voltages, suggesting the possibility of coherent spin manipulation via geometrically controlled SOIs.

\section{Results}

Sample design and measurement principle. We used two-dimensional electron systems in (001) III-V semiconductor quantum wells (QWs), which provide SOIs suited to the direct observation of a PSH. The $k$-linear term of the SOI Hamiltonian for this system is written as $H_{\mathrm{SO}}=(\alpha-\beta) k_{y} \sigma_{x}-(\alpha+\beta) k_{x} \sigma_{y}$, using a coordinate system with base vectors $x\|[110], y\|[110]$ and $z \|[001]$. The parameters $\alpha$ and $\beta$ represent the strengths of Rashba and Dresselhaus SOIs, respectively. In the balanced condition where $\alpha=\beta(\alpha=-\beta)$, the effective magnetic fields become unidirectional along $y(x)$ and proportional to $k_{x}\left(k_{y}\right)$, respectively. This results in the appearance of a helical spin mode or PSH that is robust against spin-independent scattering. An optical technique using the magneto-optic effect enables us to access the helical mode through the distribution of the $z$-component of the spins, which forms stripe patterns in a two-dimensional plane ${ }^{9,10}$.

To investigate the drift transport of helical spin states, we designed two samples, each of which contains a single GaAs/ AlGaAs QW ( $d_{\mathrm{QW}}$ thicknesses of 15 and $\left.25 \mathrm{~nm}\right)$ embedded in a high electron mobility transistor structure. The energy band structures calculated by the $k \cdot p$ perturbation theory ${ }^{13}$ allowed us to estimate the strengths of $\alpha$ and $\beta$, which are close to the PSH condition $(\alpha \sim \beta)$ for the $25-\mathrm{nm}$-thick QW, whereas they are far from the PSH condition $(\alpha<\beta)$ for the 15-nm-thick QW. The samples were grown by molecular-beam epitaxy and fabricated into cross-shaped mesa structures with four ohmic contacts (Fig. 1a). The samples were covered with semi-transparent $\mathrm{Au}$ gate electrodes. The voltage applied to the ohmic contacts $\left(V_{x}\right.$ or $\left.V_{y}\right)$ creates electric fields to transport electrons in the QW plane, while the top gate voltage $\left(V_{\mathrm{g}}\right)$ allows us to tune the Rashba SOI strength simultaneously $y^{5}$.

We employed Kerr microscopy to measure the spin dynamics of drifting electrons. A circularly polarized pump light from a continuous wave $(\mathrm{cw})$ Ti:sapphire laser generates electron spins oriented in the $z$ direction at a certain position defined as $(x, y)=(0,0)$ on the QW, and a linearly polarized light probes the magneto-optic Kerr rotation $\theta_{\mathrm{K}}$, which is proportional to the spin density at the focused position. For the microscopic measurement of spin coherence, the pump and probe lights were focused on the sample with 6 and $3 \mu \mathrm{m}$ diameter spots, which are smaller than the typical spin precession length resulting from an SOI $(\sim 20 \mu \mathrm{m})$ estimated for the present QWs.

Kerr imaging of drift spin transport. The application of an inplane electric field changes the spin transport regime significantly. Figure $1 \mathrm{~b}$ shows a one-dimensional scan of $\theta_{\mathrm{K}}$ along the $x$-axis measured for different in-plane voltages $V_{x}$ and a fixed $V_{\mathrm{g}}$ of $-4.45 \mathrm{~V}$ applied to the 25-nm-thick QW. The symmetrical

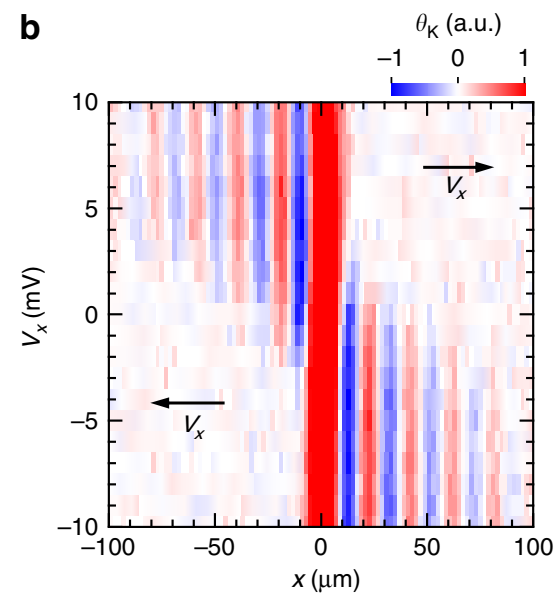

Figure 1 | Creation of drift spin flows in a cross-shaped device. (a) Schematic of top view of our samples. (b) In-plane electric field dependence of spin distribution in steady state in 25-nm-thick QW at $V_{\mathrm{g}}=-4.45 \mathrm{~V}$. 
diffusion of the spins at $V_{x}=0 \mathrm{~V}$ shows the spin precession caused by the SOI, as reported previously ${ }^{9}$, but it only appears in a small area $|x| \lesssim 10 \mu \mathrm{m}$. When we increase $\left|V_{x}\right|$, the signal becomes asymmetric, indicating that spins flow in the electron drift direction. The Kerr signals observed under in-plane electric fields provided real-space imaging of the drifting spins in a steady state, whose dynamics have been previously investigated in QWs only with a Dresselhaus SOI by spin grating spectroscopy ${ }^{11,12}$. The oscillating signal of drifting spins is maintained for a much longer distance than at $V_{x}=0 \mathrm{~V}$, thus enabling us to determine the SOI strengths more precisely.

The effects induced by the coexistence of the Rashba and Dresselhaus SOIs appear as anisotropy of the spatial precession frequency for different moving directions. Figure $2 a-c$ maps the spatial distribution of electron spins drifting in the [1 $1 \overline{1} 0]$ and [110] directions as well as that of spins without drift motion. In the 15-nm-thick QW under $V_{\mathrm{g}}=-5.0 \mathrm{~V}$ (Fig. 2a), the precessions have different spatial frequencies for the $x$ and $y$ directions. This is attributed to the anisotropic effective magnetic fields, which are expected to be proportional to $|\alpha+\beta|$ for $k_{x}$ and $|\alpha-\beta|$ for $k_{y}$. Because of the moderate anisotropy of the effective magnetic field in the $15-\mathrm{nm}$-thick $\mathrm{QW}$, the $\theta_{\mathrm{K}}$ oscillations caused by the spin precession have several periods in both directions. On the other hand, in the 25-nm-thick QW (Fig. 2b,c) the extreme anisotropy has a large effect on the spatial precession frequency, namely the precessions of spins drifting along the $y$-axis slow almost to a halt.
Such a slow precession cannot be resolved by the stripe patterns in the small region observed in the regime where no in-plane electric field is applied ${ }^{9}$. Instead, the extended transport length for drifting spins along $y$ reveals a rotation of more than $\pi$ caused by a small effective magnetic field. By carefully tuning $V_{\mathrm{g}}$ from $-4.40 \mathrm{~V}$ (Fig. 2b) to $-4.28 \mathrm{~V}$, (Fig. 2c), we achieved a condition where the spin precession was halted completely even in the drifting regime. This indicates the uniaxial configuration of the effective magnetic fields, which provide the exact SU(2) symmetry with great accuracy.

For a quantitative analysis of the spin dynamics during drift transport, we extracted two parameters that characterize the two-dimensional distribution of the Kerr rotation signal. We fitted the cross-sectional profile of the data with a function $\theta_{\mathrm{K}}(d)=\exp \left(-d / l_{\mathrm{s}}\right) \cos \left(2 \pi d / L_{\mathrm{SO}}\right)$, where $d$ is the distance from the origin, $l_{s}$ is the spin decay length caused by the spin diffusion and/or decoherence, and $L_{\mathrm{SO}}$ is the spin precession length in which electron spins rotate a full cycle. As shown in Fig. $2 a-c$, the data are fitted well and allow us to obtain the SOI parameters by using the following relations:

$$
\begin{aligned}
& \alpha=\frac{\pi \hbar^{2}}{2 m^{*}}\left(\frac{1}{L_{\mathrm{SO}}^{[110]}}+\frac{1}{L_{\mathrm{SO}}^{[110]}}\right), \\
& \beta=\frac{\pi \hbar^{2}}{2 m^{*}}\left(\frac{1}{L_{\mathrm{SO}}^{[110]}}-\frac{1}{L_{\mathrm{SO}}^{[110]}}\right),
\end{aligned}
$$

a

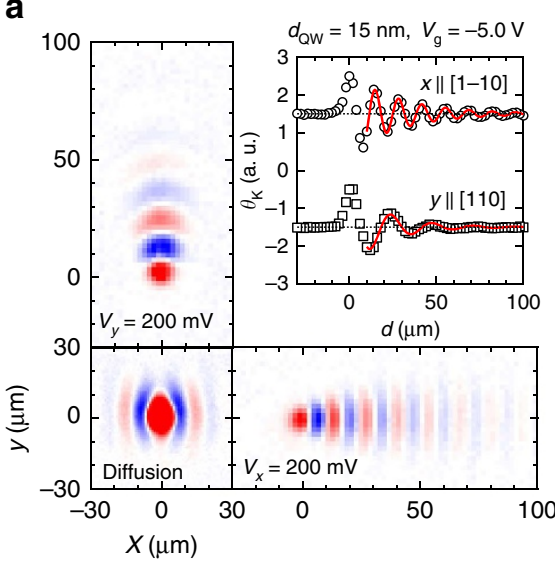

b

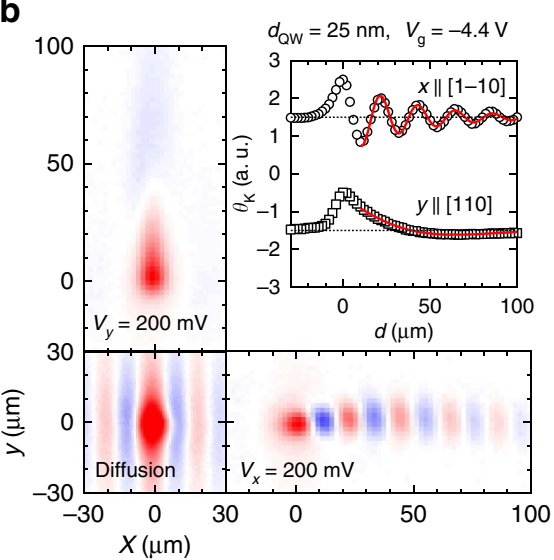

C
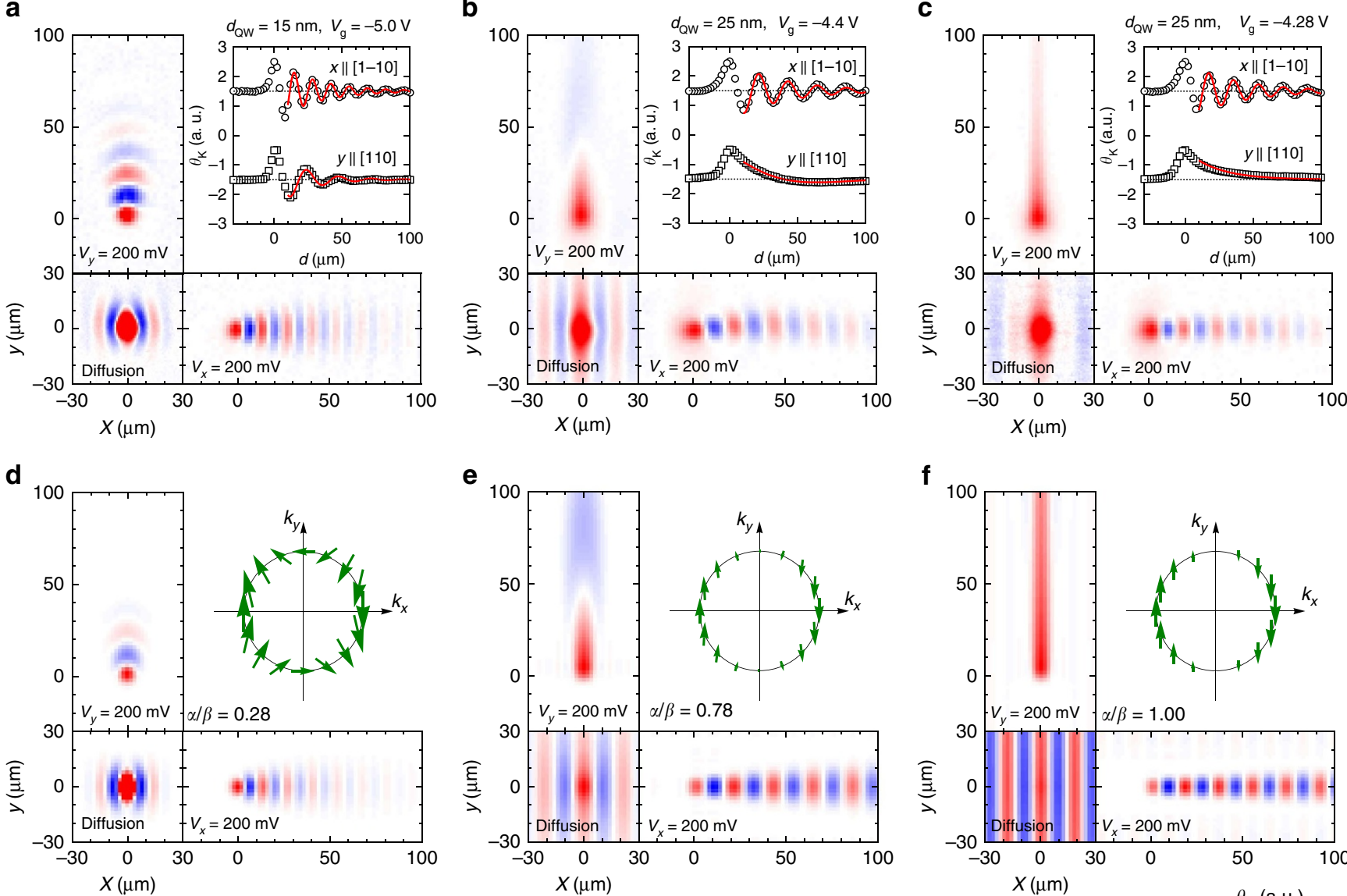

e

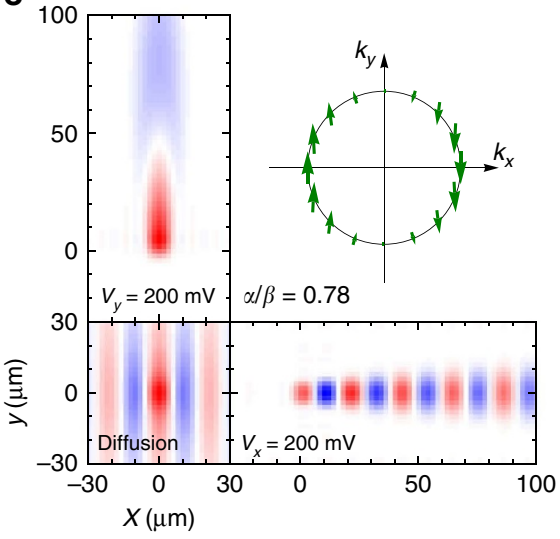

f

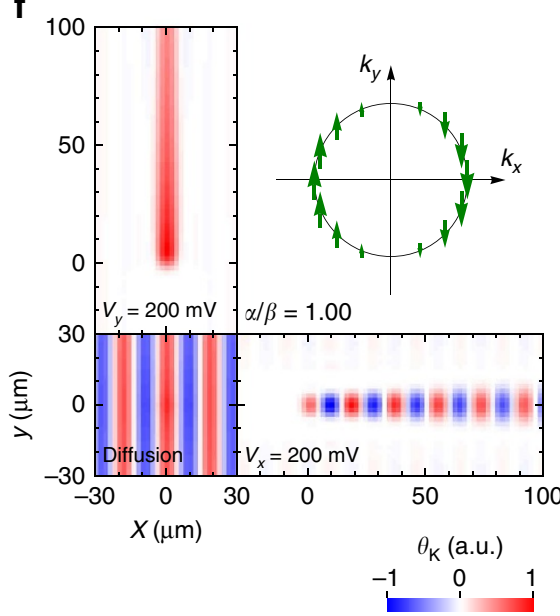

Figure 2 | Mapping of spins with/without in-plane electric fields. (a-c) Kerr rotation image of spins with/without in-plane electric fields in 15-nm-thick QW at $V_{g}=-5.0 \mathrm{~V}(\mathbf{a})$, and 25-nm-thick QW at $V_{\mathrm{g}}=-4.4 \mathrm{~V}(\mathbf{b})$ and $-4.28 \mathrm{~V}$ (c). Insets are cross-sectional patterns of drifting spins in the $x$ and $y$ directions. (d-f) Spatial mapping of drifting spins simulated by SDD model, corresponding to a-f. Insets show effective magnetic fields in $k$-space for given SOI parameters. 
where $\hbar$ and $m^{*}$ are Planck's constant and the effective mass of an electron, respectively. The $L_{\mathrm{SO}}$ obtained by the fitting and SOI parameters calculated from Equations (1) and (2) are summarized in Table 1 . We confirmed that $\alpha$ is tuned by the gate voltage and has the same value as $\beta$ at $V_{\mathrm{g}}=-4.28 \mathrm{~V}$. We also found that there was a slight variation in $\beta=\gamma<k_{z}^{2}>$, where $\gamma$ is a material constant, when modifying the gate voltage, which is due to the gate voltage dependence of the wavenumber $\left\langle k_{z}^{2}>\right.$ quantized in the $z$-direction.

Theoretical simulation with spin drift-diffusion model. The spatial evolution of spins observed in Fig. 2a-c shows excellent agreement with a theoretical calculation (Fig. 2d-f) performed using a spin drift-diffusion (SDD) equation:

$$
\frac{\partial s_{i}}{\partial t}=D_{s} \tilde{\nabla}^{2} s_{i}+(\mathbf{v} \cdot \tilde{\nabla}) s_{i},
$$

where $s_{i}$ is the $i$-th component of the spin vector, $D_{\mathrm{s}}$ is the spin diffusion coefficient, $\mathbf{v}$ is the drift velocity, and $\tilde{\nabla}$ is the covariant derivative in a system treated with a spin-dependent $S U(2)$ vector potential ${ }^{14}$. We transformed Equation (3) to the Fourier space and obtained the equation:

$$
\hat{\mathbf{g}}_{z}=\left\{D_{s}\left(q_{x}^{2}+q_{y}^{2}\right) \mathbf{E}+i\left(q_{x} v_{x}+q_{y} v_{y}\right) \mathbf{E}+\tilde{\mathbf{D}}\right\} \hat{\boldsymbol{\rho}},
$$

where $q_{i}$ is the wavenumber of the spin mode in the $x$ and $y$ directions and $\mathbf{E}$ is an identical matrix. $\hat{\boldsymbol{\rho}}=\left(\hat{\rho}_{x}, \hat{\rho}_{y}, \hat{\rho}_{z}\right)$ and $\hat{\mathbf{g}}_{z}=\left(0,0, \hat{g}_{z}\right)$ are the spin polarization and generation matrix in the Fourier space, respectively. We used a two-dimensional Gauss

\begin{tabular}{|c|c|c|c|}
\hline $\begin{array}{l}d_{\mathrm{QW}}(\mathrm{nm}) \\
V_{\mathrm{g}}(\mathrm{V})\end{array}$ & $\begin{array}{c}15 \\
-5.0 \\
\end{array}$ & $\begin{array}{c}25 \\
-4.4 \\
\end{array}$ & $\begin{array}{c}25 \\
-4.28 \\
\end{array}$ \\
\hline$D_{s}\left(\mathrm{~cm}^{2} \mathrm{~s}^{-1}\right)$ & $60.8 \pm 10.1$ & $40.8 \pm 9.2$ & $17.6 \pm 8.7$ \\
\hline$l_{s}^{[1-10]}(\mu \mathrm{m})$ & $29.6 \pm 4.0$ & $37.8 \pm 6.6$ & $37.5 \pm 5.9$ \\
\hline$I_{s}^{[110]}(\mu \mathrm{m})$ & $21.2 \pm 3.4$ & $35.0 \pm 4.2$ & $23.2 \pm 5.2$ \\
\hline$L_{S O}^{[1-10]}(\mu \mathrm{m})$ & $13.5 \pm 0.2$ & $21.5 \pm 0.7$ & $18.3 \pm 0.4$ \\
\hline$L_{S O}^{[110]}(\mu \mathrm{m})$ & $23.7 \pm 1.5$ & $172.9 \pm 15.7$ & - \\
\hline$\alpha(\mathrm{meV} \AA)$ & $0.58 \pm 0.04$ & $0.74 \pm 0.07$ & $0.99 \pm 0.02$ \\
\hline$\beta(\mathrm{meV} \AA)$ & $2.10 \pm 0.13$ & $0.95 \pm 0.09$ & $0.99 \pm 0.02$ \\
\hline
\end{tabular}
function with a $6 \mu \mathrm{m}$ half width at half maximum for the steady

The parameters $\left(D_{\mathrm{s}}, I_{\mathrm{s}}, L_{\mathrm{SO}}, \alpha\right.$ and $\left.\beta\right)$ and their uncertainties (s.d.) for different $d_{\mathrm{QW}}$ and $V_{\mathrm{g}}$ values are derived by a least-squares fit. generation of spin polarization. The first and second terms in Equation (4) represent the spin diffusion and drift transport of spins, respectively, whereas $\tilde{\mathbf{D}}$ is a matrix including the spin rotation and relaxation given by,

$$
\tilde{\mathbf{D}}=\left(\begin{array}{ccc}
-D_{\mathrm{s}} q_{1}^{2} & 0 & q_{1}\left(i 2 D_{\mathrm{s}} q_{x}+v_{x}\right) \\
0 & -D_{\mathrm{s}} q_{2}^{2} & q_{2}\left(i 2 D_{\mathrm{s}} q_{y}+v_{y}\right) \\
-q_{1}\left(i 2 D_{\mathrm{s}} q_{x}+v_{x}\right) & -q_{2}\left(i 2 D_{\mathrm{s}} q_{y}+v_{y}\right) & -D_{s}\left(q_{1}^{2}+q_{2}^{2}\right)
\end{array}\right)
$$

where $q_{1}=2 m^{*}(\alpha+\beta) / \hbar^{2}$ and $q_{2}=2 m^{*}(\alpha-\beta) / \hbar^{2}$. The spin distribution in real space can be obtained from the inverse Fourier transform of the solution of Equation (4). The contribution of the cubic Dresselhaus term is neglected in the simulation because the Fermi wavenumber $k_{\mathrm{F}}$ and drift wavenumber $k_{\mathrm{d}}=m^{*} v_{\mathrm{d}} / \hbar$ are estimated to be $10^{7} \mathrm{~m}^{-1}$ or smaller for the condition used in the experiment, thus the cubic term $\left(\Omega_{\text {cubic }}=\gamma k^{3} / \hbar\right)$ should be one or two orders smaller than the linear term $\left(\Omega_{\text {linear }}=2 \beta k / \hbar\right)$. The $D_{\text {s }}$ values were estimated from the lateral broadening of the Kerr rotation signal for the spins drifting in the $x$ direction (shown in Table 1), and the $\mathbf{v}$ values were obtained from a time-resolved measurement of the spin transport in the same samples. The calculated results shown in Fig. 2d-f well reproduce all the spin dynamics behaviours including the precession, expansion, and decay of the drifting and diffusing spins.

Our experimental and calculated results show that the $\mathrm{PSH}$ condition is favourable for long-distance drift spin transport, which agrees with the prediction by random walk theory ${ }^{15}$. Figure 3a shows the result of an SDD simulation, in which the $s_{z}$ values are plotted as a function of $x$ and $\alpha$ for the drift spins moving in the $x$ direction. Because we fixed the $\beta$ value in the calculation, we can expect the spatial precession frequency $\left(1 / L_{\mathrm{SO}}^{[1 \overline{1} 0]}\right)$ to increase linearly with $\alpha$, according to Equations (1) and (2). Similar behaviour was observed experimentally for drifting spins around the PSH condition via the gate control of the Rashba SOI (Fig. 3b). Assuming that the precession period in Fig. $3 b$ depends only on $\alpha$, we can compare the effect of $\alpha$ on the transport length by plotting $l_{\mathrm{s}}$ versus $L_{\mathrm{SO}}$, where both parameters can be extracted by the fitting procedure used in Fig. 2. The obtained $l_{\mathrm{s}}$ values are plotted as a function of $L_{\mathrm{SO}}$ in Fig. 3c. In both the experiment and the simulation, $l_{\mathrm{s}}$ decreases as $L_{\mathrm{SO}}$ diverges from the balanced condition $\alpha=\beta$, indicating that the suppression of the D'yakonov- Perel' (DP) spin relaxation caused by PSH also appears in the spin transport length. It should be noted that the fact that the spin decay length in the experiment is shorter than in the simulation could be due to spin decoherence
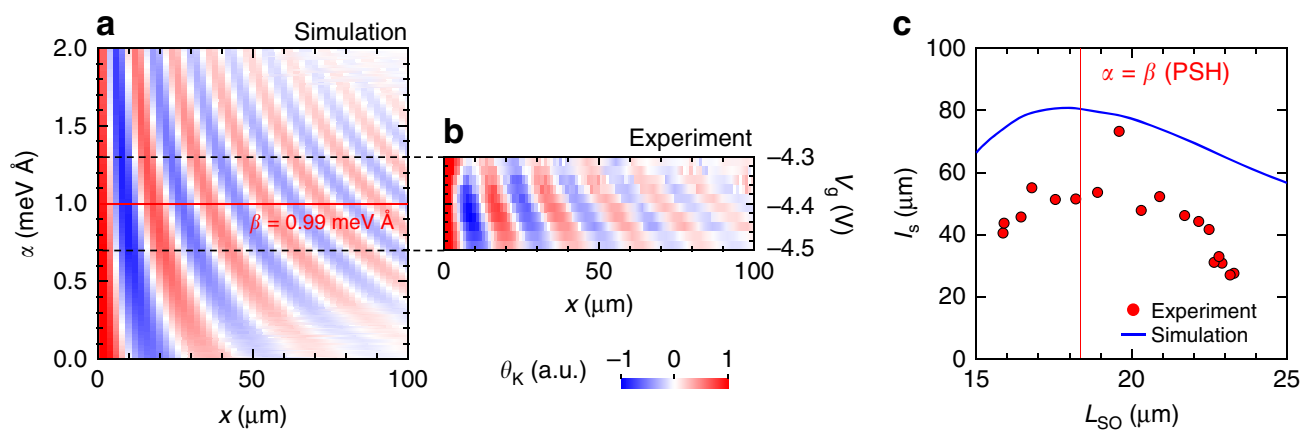

Figure 3 | SOI dependence of spin dynamics of drifting spins. (a) Comparison of simulated and experimental spin distribution driven by $V_{x}=50 \mathrm{~m} V$ in the $x$ direction. In the simulation, we directly varied the Rashba SOI parameters $\alpha$ and the spin distribution is shown as a function of $\alpha$, whereas (b) the experimental data are plotted as a function of gate voltage, which modulates the Rashba SOI. Note that the gate voltage shown in this figure does not correspond to that in other experiments because the measurements were carried out on different days leading to different offset voltages. (c) Spin decay length as a function of spin precession length. 


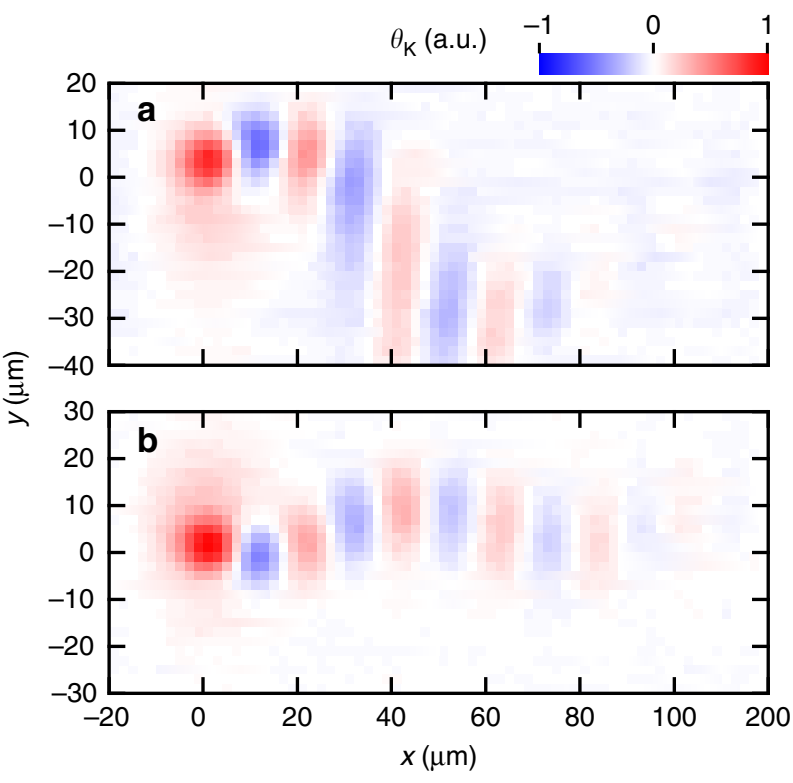

Figure 4 | Dynamic modulation of transport path of drifting spins.

$(\mathbf{a}, \mathbf{b})$ Drift spin transport driven by $V_{x}^{\mathrm{dc}}=100 \mathrm{mV}$ with sinusoidal voltage $V_{y}^{\mathrm{ac}}=200 \mathrm{mV}$ applied in the $x$ and $y$ direction, respectively. The frequency of the $V_{y}^{\mathrm{ac}}$ was adjusted to the repetition frequency of the mode-locked laser, $82.46 \mathrm{MHz}$ (a) or twice the frequency (b).

caused by the cubic Dresselhaus term although its magnitude is one order smaller than the linear Dresselhaus term.

Modulation of spin transport path by time-varying electric fields. We can transport the helical spin state along arbitrary trajectories by using a time-dependent in-plane electric field. We applied a sinusoidal ac voltage $V_{y}^{\text {ac }}$ with frequency $f$ in the $y$ direction and dc voltages $V_{x}^{\mathrm{dc}}$ in the $x$ direction. To excite spins at the timing of a certain phase of $V_{y}^{\text {ac }}$, the initial spins were excited with a pulsed pump light from a mode-locked laser whose repetition frequency was synchronized with $f$ or $f / 2$, whereas the Kerr signal was detected with a probe light from a cw laser. When a sinusoidal voltage was applied in the transverse direction of the drift spin transport, a clear spin flow along a winding path was observed (Fig. 4). The stripe patterns in the spin packet are maintained regardless of transport trajectories. This is because, in a PSH condition, spins always precess in an $x-z$ plane and the precession phase depends only on $x$. For all the transport paths, the spin phase was conserved even at distances much longer than the precession periods, and thus the technique will be beneficial for further exploitation of the SOI in drift spin manipulation.

The results presented here show that spin coherence with a balanced SOI can be efficiently transferred to a distant place without the aid of any static narrow quasi-one-dimensional channels. Because we can use lateral electric fields for highmobility electron systems, the transport speed can be much faster than that in bulk systems ${ }^{16,17}$ or acoustic waves ${ }^{18-20}$, which have been used in previous spin-transport experiments. If the balanced condition is sufficiently maintained, we may also manipulate the spin states by using the gate bias voltage or trajectory-controlled quantum operation ${ }^{19,21}$. Further development of this technique will advance the field of semiconductor spintronics including the physics of a spin-charge coupled system 22,23 as well as applications for future spin devices $6,24,25$.

\section{Methods}

Sample fabrication. The samples were 15- and 25-nm-thick modulation-doped $\mathrm{Al}_{0.3} \mathrm{Ga}_{0.7} \mathrm{As} / \mathrm{GaAs} / \mathrm{Al}_{0.3} \mathrm{Ga}_{0.7}$ As single $\mathrm{QW}$ grown by molecular-beam epitaxy on a (001) semi-insulating GaAs substrate. The QW was located $120 \mathrm{~nm}$ below the surface, whereas three $\delta$-doping layers were embedded 50, 100 and $105 \mathrm{~nm}$ below the surface. The epitaxial wafers were fabricated into cross-shaped structures with four ohmic contacts consisting of AuGe $(260 \mathrm{~nm})$ and $\mathrm{Ni}(40 \mathrm{~nm})$ layers. The central part of the structure was covered with a 7-nm-thick Au gate electrode through which we could obtain the optical signal from the QW.

Optical measurement. We employed spatially resolved Kerr rotation microscopy in which spin polarization along the $z \|[001]$ direction was generated with a circularly polarized pump light $(100 \sim 275 \mu \mathrm{W}$ average power) from a cW Ti:sapphire laser and detected with a linearly polarized probe light $(1.0 \sim 6.8 \mu \mathrm{W})$. To excite spins at the timing of a certain phase of $V_{y}^{\text {ac }}$, we undertook an experiment involving drifting spin path modulation induced by time-dependent in-plane electric fields. The initial spins were excited with a pulsed pump light from a modelocked laser whose repetition frequency was synchronized with the frequency $f$ of the in-plane electric field, whereas the Kerr signal was detected with a probe light from a cw laser. The wavelengths of both the pump and probe lights were adjusted to maximize the Kerr signals for each QW and gate voltage. The polarization of the pump light was modulated between left- and right-circular polarizations at $50.1 \mathrm{kHz}$, and the probe light was chopped with an acousto-optic modulator at $52.0 \mathrm{kHz}$. The difference frequency $(1.9 \mathrm{kHz})$ was used as a reference for lock-in detection. The full width at half maximum spot size of the normally incident probe beam was $\sim 3 \mu \mathrm{m}$, whereas the waist size of the obliquely incident pump beam was $6 \mu \mathrm{m}$ and its spot on the sample was slightly elongated in the [1 10$]$ direction. The position of the probe light spot was scanned in the QW plane for spatially resolved Kerr rotation measurements. All the measurements were carried out at $8 \mathrm{~K}$.

\section{References}

1. Datta, S. \& Das, B. Electronic analog of the electro-optic modulator. Appl. Phys. Lett. 56, 665-667 (1990).

2. Wolf, S. A. et al. Spintronics: A spin-based electronics vision for the future. Science 294, 1488-1495 (2001).

3. Wunderlich, J. et al. Spin Hall effect transistor. Science 330, 1801-1804 (2010).

4. D'yakonov, M. I. \& Perel', V. I. Spin relaxation of conduction electrons in noncentrosymmetric semiconductors. Sov. Phys. Solid State 13, 3023-3026 (1972).

5. Nitta, J., Akazaki, T., Takayanagi, H. \& Enoki, T. Gate control of spin-orbit interaction in an inverted $\mathrm{In}_{0.53} \mathrm{Ga}_{0.47} \mathrm{As} / \mathrm{In}_{0.52} \mathrm{Al}_{0.48}$ As heterostructure. Phys. Rev. Lett. 78, 1335-1338 (1997).

6. Schliemann, J., Egues, J. C. \& Loss, D. Nonballistic spin-field-effect transistor. Phys. Rev. Lett. 90, 146801 (2003).

7. Bernevig, B. A., Orenstein, J. \& Zhang, S.-C. Exact SU(2) symmetry and persistent spin helix in a spin-orbit coupled system. Phys. Rev. Lett. 97, 236601 (2006).

8. Koralek, J. D. et al. Emergence of the persistent spin helix in semiconductor quantum wells. Nature 458, 610-613 (2009).

9. Walser, M. P., Reichl, C., Wegscheider, W. \& Salis, G. Direct mapping of the formation of a persistent spin helix. Nat. Phys. 8, 757-762 (2012).

10. Ishihara, J., Ohno, Y. \& Ohno, H. Direct imaging of gate-controlled persistent spin helix state in a modulation-doped GaAs/AlGaAs quantum well. Appl. Phys. Exp. 7, 013001 (2014).

11. Yang, L. et al. Doppler velocimetry of spin propagation in a two-dimensional electron gas. Nat. Phys. 8, 153-157 (2012).

12. Yang, L. et al. Coherent propagation of spin helices in a quantum well confined electron gas. Phys. Rev. Lett. 109, 246603 (2012).

13. Engels, G., Lange, J., Schäpers, T. \& Lüth, H. Experimental and theoretical approach to spin splitting in modulation-doped $\operatorname{In}_{x} \mathrm{Ga}_{1-x} \mathrm{As} / \mathrm{InP}$ quantum wells for B $\rightarrow 0$. Phys. Rev. B 55, R1958-R1961 (1997).

14. Gorini, C., Schwab, P., Raimondi, R. \& Shelankov, A. L. Non-Abelian gauge fields in the gradient expansion: Generalized Boltzmann and Eilenberger equations. Phys. Rev. B 82, 195316 (2010).

15. Yang, L., Orenstein, J. \& Lee, D.-H. Random walk approach to spin dynamics in a two-dimensional electron gas with spin-orbit coupling. Phys. Rev. B 82, 155324 (2010).

16. Kato, Y. K., Myers, R. C., Gossard, A. C. \& Awschalom, D. D. Observation of the spin Hall effect in semiconductors. Science 306, 1910-1913 (2004).

17. Kikkawa, J. M. \& Awschalom, D. D. Lateral drag of spin coherence in gallium arsenide. Nature 397, 139-141 (1999).

18. Stotz, J. A. H., Hey, R., Santos, P. V. \& Ploog, K. H. Coherent spin transport through dynamic quantum dots. Nat. Mater. 4, 585-588 (2005).

19. Sanada, H. et al. Manipulation of mobile spin coherence using magnetic-fieldfree electron spin resonance. Nat. Phys. 9, 280-283 (2013).

20. Sogawa, T. et al. Transport and lifetime enhancement of photoexcited spins in GaAs by surface acoustic waves. Phys. Rev. Lett. 87, 276601 (2001).

21. Golovach, V. N., Borhani, M. \& Loss, D. Holonomic quantum computation with electron spins in quantum dots. Phys. Rev. A 81, 022315 (2010). 
22. Mishchenko, E. G., Shytov, A. V. \& Halperin, B. I. Spin current and polarization in impure two-dimensional electron systems with spin-orbit coupling. Phys. Rev. Lett. 93, 226602 (2004).

23. Raimondi, R., Gorini, C., Schwab, P. \& Dzierzawa, M. Quasiclassical approach to the spin Hall effect in the two-dimensional electron gas. Phys. Rev. B 74, 035340 (2006).

24. Ohno, M. \& Yoh, K. Datta-Das-type spin-field-effect transistor in the nonballistic regime. Phys. Rev. B 77, 045323 (2008).

25. Kunihashi, Y. et al. Proposal of spin complementary field effect transistor. Appl. Phys. Lett. 100, 113502 (2012).

\section{Acknowledgements}

We thank K. Sasaki and P.V. Santos for useful discussions. This work received support from the Japan Society for the Promotion of Science (JSPS) KAKENHI Grant Number 15H05699.

\section{Author contributions}

Y.K., H.S., H.G. and T.S. designed the experiments; K.O. grew the sample; Y.K. and H.S. performed the measurements; M.K. and J.N. gave theoretical support and conceptual advice; Y.K. wrote the manuscript; and all the authors discussed the results and commented on the manuscript at all stages.

\section{Additional information}

Competing financial interests: The authors declare no competing financial interests.

Reprints and permission information is available online at http://npg.nature.com/ reprintsandpermissions/

How to cite this article: Kunihashi, Y. et al. Drift transport of helical spin coherence with tailored spin-orbit interactions. Nat. Commun. 7:10722 doi: 10.1038/ncomms10722 (2016).

\section{(c) (i)}

This work is licensed under a Creative Commons Attribution 4.0 International License. The images or other third party material in this article are included in the article's Creative Commons license, unless indicated otherwise in the credit line; if the material is not included under the Creative Commons license, users will need to obtain permission from the license holder to reproduce the material. To view a copy of this license, visit http://creativecommons.org/licenses/by/4.0/ 